\title{
Combining Transcranial Magnetic Stimulation and Electroencephalography May Contribute to Assess the Severity of Alzheimer's Disease
}

\author{
Petro Julkunen, ${ }^{1}$ Anne M. Jauhiainen, ${ }^{2}$ Mervi Könönen,, ${ }^{1,}$ Ari Pääkkönen, ${ }^{1}$ \\ Jari Karhu, ${ }^{4,5}$ and Hilkka Soininen ${ }^{2,6}$ \\ ${ }^{1}$ Department of Clinical Neurophysiology, Kuopio University Hospital, POB 1777, 70211 Kuopio, Finland \\ ${ }^{2}$ Department of Neurology, Institute of Clinical Medicine, University of Eastern Finland, 70211 Kuopio, Finland \\ ${ }^{3}$ Department of Clinical Radiology, Kuopio University Hospital, 70211 Kuopio, Finland \\ ${ }^{4}$ Nexstim Ltd, 00510 Helsinki, Finland \\ ${ }^{5}$ Department of Physiology, Institute of Biomedicine, University of Eastern Finland, 70211 Kuopio, Finland \\ ${ }^{6}$ Department of Neurology, Kuopio University Hospital, 70211 Kuopio, Finland
}

Correspondence should be addressed to Petro Julkunen, petro.julkunen@kuh.fi

Received 30 December 2010; Revised 3 March 2011; Accepted 13 March 2011

Academic Editor: Fabio Ferrarelli

Copyright $@ 2011$ Petro Julkunen et al. This is an open access article distributed under the Creative Commons Attribution License, which permits unrestricted use, distribution, and reproduction in any medium, provided the original work is properly cited.

\begin{abstract}
Alzheimer's disease (AD) is the most common form of old age dementia, and mild cognitive impairment (MCI) often precedes AD. In our previous study (Julkunen et al. 2008), we found that the combination of transcranial magnetic stimulation (TMS) and electroencephalography (EEG) was able to find distinct differences in $\mathrm{AD}$ and MCI patients as compared to controls. Here, we reanalyzed the small sample data from our previous study with the aim to test the sensitivity of the TMS-EEG characteristics to discriminate control subjects $(n=4)$ from $\mathrm{MCI}(n=5)$ and $\mathrm{AD}(n=5)$ subjects. Furthermore, we investigated how the TMS-EEG response characteristics related to the scores of the dementia rating scales used to evaluate the severity of cognitive decline in these subjects. We found that the TMS-EEG response P30 amplitude correlated with cognitive decline and showed good specificity and sensitivity in identifying healthy subjects from those with MCI or AD. Given the small sample size, further studies may be needed to confirm the results.
\end{abstract}

\section{Introduction}

Alzheimer's disease (AD) is a neurodegenerative disorder which leads to dementia through a progressive cognitive decline. In Europe, $\mathrm{AD}$ affects over $5 \%$ of population aged above 70 years [1]. This makes it the most common cause of dementia in old age. It has been postulated that the impairment of the lateral cholinergic pathway originating from the Meynert's nucleus would characterize $\mathrm{AD}$ and contributes to its typical symptom of memory loss $[2,3]$. AD-related pathology leads to the degeneration of the large cortical pyramidal neurons [4], and subsequently impairment of functional connectivity takes place [5]. Before the diagnosis of $\mathrm{AD}$ can be set, subjects often suffer from impaired episodic memory [6]. The stage characterised by mild memory or other cognitive loss is called mild cognitive impairment
(MCI), and it has been proposed as a prodromal state of AD. Thus, subjects with MCI have an increased risk to develop $\mathrm{AD}$ [7-9]. Understanding the pathophysiology of MCI would be essential for predicting and possibly in the future preventing the development of AD. It is possible that altered functional connectivity precedes structural changes, and therefore, a sensitive method to detect those early functional changes would be useful in the diagnostics of MCI and $\mathrm{AD}$. Early identification of AD would be desirable, as it could help aiming the current treatment to the appropriate subjects. With the prospects of obtaining treatments that modify the course of $\mathrm{AD}$, accurate identification of subjects who will develop $\mathrm{AD}$ is essential.

Earlier it has been shown that the primary motor cortex experiences changes during the development of $\mathrm{AD}$, which also relate to the severity of the disease [10]. Structural 
changes in M1 are mild and appear late as compared to other brain areas, and therefore, motor function also appears intact in early AD [11-14]. Several earlier TMS studies have found that $\mathrm{AD}$ patients have reduced resting motor threshold (MT) of the primary motor cortex [3, 15-21]. Alagona et al. reported that the resting MT correlates inversely with the disease severity [15]. This implies that the inhibitory control is reduced in $\mathrm{AD}$, which is also supported by reported shortening of cortical silent period [21]. Additionally, previous studies have reported reduction in short-latency afferent inhibition (SAI) in $\mathrm{AD}[18,22-24]$. SAI has been considered as a marker of central cholinergic activity [25] and is likely of cortical origin [26, 27]. Hence, motor cortex functions, especially intracortical inhibition, suffer during the development of AD. Earlier, Sakuma et al. [23] showed that SAI is not impaired in MCI, suggesting that the cholinergic activity shown to be impaired in AD may still be normal in MCI. Several studies have been conducted to solve this question and supporting as well as contradicting results have been published [28-31]. Hence, the cholinergic changes related to MCI should be interpreted carefully, as the cholinergic regulation in MCI is still unclear. Furthermore, in $\mathrm{AD}$, there is a tendency towards a reduced short-latency intracortical inhibition (SICI), a different form of inhibition evoked by using paired-pulse TMS $[3,18,19]$. SICI has been connected with intracortical $\mathrm{GABA}_{\mathrm{A}}$ activity [32].

Combining TMS with electroencephalography (EEG) offers a direct noninvasive method to study cortical reactivity and connectivity in physiological and pathological conditions [33-38]. Previously, we have shown that TMS-EEG can reveal abnormalities in functional cortical connectivity and reactivity in the $\mathrm{AD}$ subjects [39]. Our main finding was that the P30 response of TMS-EEG was significantly reduced in $\mathrm{AD}$ as compared to controls and MCI, and that the reduction was localized to the ipsilateral temporoparietal area as well as contralateral frontocentral area, that is, sensorimotor area, connected to M1. In the past, TMS-EEG response, when focused on M1, has been shown to exhibit several distinguishable peaks: N15, P30, N40, P60, and N100 [33, $35,36,38,40-43]$. Prior studies have related the early peaks $\mathrm{N} 15$ and $\mathrm{P} 30$ to the M1 activation. P30 has been suggested to reflect activity around the premotor cortex on the stimulated side, and it has been reported that P30 may increase due to long-term potentiation induced by repetitive TMS [41]. Furthermore, P30 has been suggested to involve pathways between subcortical structures such as thalamic nuclei or basal ganglia and cortex [40]. Also, P30 has been shown to vanish with nonoptimal orientation of the stimulation coil in respect to the cortical structures [40]. Therefore, the use of neuronavigation in combination with TMS allows controlling of the stimulation direction in respect to the subject's brain anatomy and results in optimized motor responses [44], and likely optimized TMS-EEG responses.

We wanted to investigate subject-specific differences in intracortical connectivity between healthy subjects, and MCI or $\mathrm{AD}$ patients. We utilized and reanalyzed our previously published data [39], which indicated that especially the P30 amplitude of the TMS-EEG response could be decreased in $\mathrm{AD}$. We further evaluated the sensitivity of the P30 amplitude changes in discriminating healthy subjects from those exhibiting cognitive impairment (MCI and AD). Furthermore, we tested whether P30 amplitude would directly relate to commonly categorizing scores of dementia rating scales. On the basis of the findings of our previous study, we hypothesized that P30 amplitude would decline as the disease becomes more severe and correlate with the dementia rating scales.

\section{Materials and Methods}

2.1. Subjects. In the present study, our previously published data was further analyzed. A small size sample including four control subjects (age: $78 \pm 3$ years, 3 females, 1 male), five MCI subjects (age: $74 \pm 8$ years, 2 females, 3 males), and five $\mathrm{AD}$ subjects (age: $73 \pm 8$ years, 2 females, 3 males) was recruited for the original study. All subjects were right handed. Each subject gave written informed consent, and the study was approved by the local ethics committee. Categorizing of these subjects to their groups was done based on a standard rating [45] and is explained in more detail in the original paper [39]. Briefly, the MCI subjects fulfilled the following characteristics [7]: (1) subjective memory impairment corroborated by an informant, (2) objective memory impairment, that is, a score of 0.5 in the clinical dementia rating (CDR) scale [45] with at least 0.5 on the memory subscale and a score of $1.5 \mathrm{SD}$ below the average of a normative age-matched sample group in at least one memory test, (3) normal global cognitive function (MiniMental-State Examination score (MMSE) of at least 20 [46]), (4) normal activities of daily living, and (5) no dementia according to the NINCDS-ADRDA criteria [11]. The MCI subjects were classified as multidomain amnestic MCI [47]. Diagnosis of $A D$ was made according to the NINCDS-ADRDA criteria for probable AD [11]. All the $\mathrm{AD}$ patients were on cholinesterase inhibitors, while other subjects had no medication affecting cognition at the time of measurements.

\subsection{Measurement System and Protocol. Navigated TMS was} used to probe the motor cortex of the subjects (Figure 1), that is, the primary motor cortex (M1) of the subjects was mapped for the representation area of the thenar musculature of both hands. The stimulation system consisted of a Magstim BiStim stimulator (Magstim Ltd., Whitland, UK) and a $70 \mathrm{~mm}$ figure-of-eight TMS coil with monophasic pulse form. Stimulation-triggered EEG responses were recorded with $1450 \mathrm{~Hz}$ sampling frequency and $16 \mathrm{bit}$ precision using a 60-channel TMS-compatible EEG amplifier (Nexstim Ltd., Helsinki, Finland). Navigation of the TMS system utilized T1-weighted 3D magnetic resonance images (imaged with Siemens Magnetom Avanto, Siemens, Erlangen, Germany). The navigation was conducted using eXimia navigation system (version 2.0, Nexstim Ltd., Helsinki, Finland). Resting state MT at the "hotspot" was determined using a threshold-hunting protocol [48]. For measuring TMS-induced muscle responses, electromyography was recorded (ME6000, Mega Electronics Inc., Kuopio, Finland) 


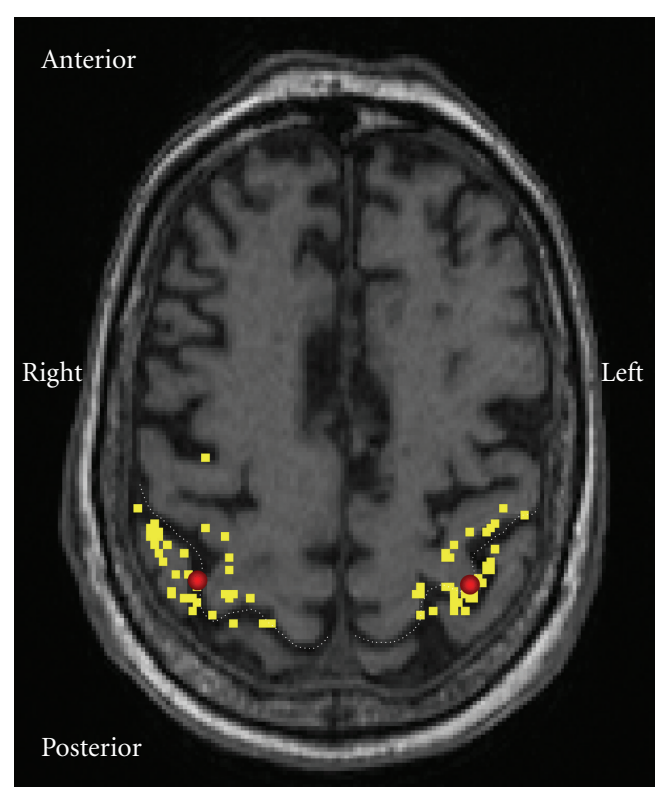

FIGURE 1: Motor cortex representation area of a control patient. TMS was focused at the "hotspot" of thenar muscle representation on the primary motor cortex (M1). The yellow dots present stimulation locations during the mapping of the hotspot in the vicinity of M1. The red spots indicate the hotspots, which were located within normal variation in each group [57].

from the opponens pollicis muscle using pregelled disposable $\mathrm{Ag}-\mathrm{AgCl}$ surface electrodes. TMS-induced EEG responses were recorded from $>50$ trials elicited with an interstimulus interval of $3-5 \mathrm{~s}$ with a stimulation intensity of $110 \%$ of the determined MT. For a more thorough system description, the reader is referred to our previous paper [39]. Both hemispheres were separately investigated, and the stimulation order of the hemispheres was randomized.

2.3. Analysis of TMS-EEG. The offline analysis of EEG was performed using Matlab 7.2 (Mathworks Inc., Natick, MA). Zero padding for $10 \mathrm{~ms}$ after the TMS pulse was applied to dampen the TMS-induced artefact. Segmented EEG was bandpass filtered to $1-50 \mathrm{~Hz}$. Any segments contaminated by blinks, as observed from vertical electro-oculogram, were removed from the analyses. Also, in some cases, a bad channel signal due to poor contact was replaced with a signal linearly interpolated from the neighbouring good channels. Manual artefact removal was conducted, prior to rereferencing all electrodes to common average. Baseline correction for $100 \mathrm{~ms}$ before each stimulus was conducted prior to averaging the segments. Averaged TMS-EEG responses over all trials were used in the statistical comparisons (Figure 2). Our interest was in the P30 response, earlier shown to be influenced by $\mathrm{AD}$ [39]. Analysis of the P30 responses was conducted from an electrode close to the site of stimulation based on the most distinguishable and shortest-latency response, as it has been reported that $\mathrm{P} 30$ originates ipsilaterally to the stimulation at the close proximity of the primary motor cortex (M1) [33].
2.4. Statistical Analyses. To test how well the P30 amplitude would be able to discriminate the groups from each other, receiver operating characteristic (ROC) curve analysis was conducted. Area under the ROC curve (AUC) was computed to determine how well the groups could be discriminated based on the P30 amplitude. The asymptotic significance for the AUC was computed with the null hypothesis of AUC = 0.5 . The optimal cut-off point for the ROC curve was determined as the closest point to the diagonal line connecting points $(0,1)$ and $(1,0)$ in the ROC plot.

Differences in P30 amplitude between the groups were analysed applying a mixed linear model, and using group and hemisphere as fixed variables and subject as a random variable. Restricted maximum likelihood estimation was used in the model. Mean effects between the groups were analysed using post-hoc analysis with least significant difference adjustment (LSD). Also, individual mean amplitudes for the P30 (hemispheric values averaged, P30 mean) were used in the comparisons. Then, Mann-Whitney test was applied in comparison of the differences between the groups. Correlations between the scores of dementia rating scales and P30 amplitude were conducted using Spearman's rank correlation $(\rho)$. The tests for correlation significance were two tailed. The correlated dementia scores were the global score and sum of boxes score of the clinical dementia rating scale (CDR-SOB) as well as MMSE. Statistical tests were conducted using SPSS 17 (SPSS Inc., Chicago, IL). Level of statistical significance was set at $P<.05$.

\section{Results}

The resting MTs (average of both hemispheres) for the opponens pollicis muscle of the control, $\mathrm{MCI}$, and $\mathrm{AD}$ group were $44 \pm 11,48 \pm 12$, and $41 \pm 4 \%$ of the maximum stimulator output, respectively. No significant differences were observed between the groups. The hemispheric data and data for pooled samples were presented in our previous study [39], where we found that the MT of the left hemisphere in MCI subjects ( $50 \pm 13 \%$ of the maximum stimulator output) was significantly higher $(P<.05)$ than in $\mathrm{AD}$ patients $(42 \pm$ $4 \%$ of the maximum stimulator output). Additionally, on the right hemisphere, the MT of the controls $(40 \pm 11 \%$ of the maximum stimulator output) was significantly lower $(P<.05)$ than in MCI subjects $(48 \pm 13 \%$ of the maximum stimulator output).

Dementia scales for the subject groups were distinctive of the different disease conditions. MMSE for control, MCI, and $\mathrm{AD}$ group was $27 \pm 4,25 \pm 3$, and $22 \pm 5$, respectively. The global CDR values were 0 for controls, 0.5 for MCI subjects, and $0.5(n=4)$ or $1.0(n=1)$ for $\mathrm{AD}$ patients. Corresponding values for the CDR-SOB were $0.0 \pm 0.0$, $1.9 \pm 1.1$, and $3.2 \pm 2.5$, respectively. CDR and CDR-SOB values in controls were significantly lower $(P<.001)$ than in $\mathrm{MCI}$ and $\mathrm{AD}$ subjects, while MMSE was nonsignificantly higher $(P=.055)$ in controls as compared to AD group. Also, no significant difference was observed between controls and MCI subjects in MMSE $(P=.437)$.

The $\mathrm{P} 30$ peak was lower in amplitude in the $\mathrm{AD}$ patients than in the controls (Figure 3, Table 1). No significant 
TABLe 1: Group-wise values of P30 amplitude.

\begin{tabular}{lccr}
\hline & & P30 amplitude $(\mu \mathrm{V})$ & AD \\
& Control & MCI & $17.7 \pm 7.1$ \\
Left hemisphere & $32.0 \pm 6.0$ & $25.6 \pm 12.7$ & $11.5 \pm 4.9$ \\
Right hemisphere & $33.0 \pm 14.6$ & $16.3 \pm 5.9$ & $16.0 \pm 6.9^{*}$ \\
P30 & $32.5 \pm 9.8$ & $21.1 \pm 8.2$ & 1.2 \\
\hline
\end{tabular}

${ }^{*} P<.05$ as compared to controls, linear mixed model (pooled values), and Mann-Whitney test (mean or hemispheric values).

Abbreviations:

MCI: Mild cognitive impairment

AD: Alzheimer's disease

P30 mean: P30 amplitude, mean of P30 amplitudes on both hemispheres.

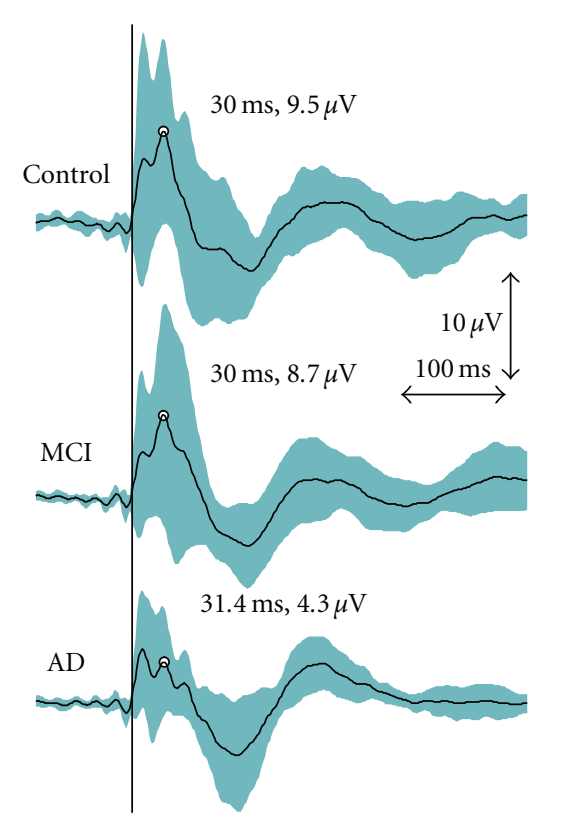

FIGURE 2: Grand average curves for TMS-evoked EEG responses as measured from the central electrode $(\mathrm{CZ})$. The mean peak for the P30 has been indicated. However, P30 was analyzed for individuals from the electrode chosen based on the shortest latency and clearest identification on the stimulated hemisphere. The turquoise area represents the 95\% confidence interval for the TMS-EEG responses. The vertical black line indicates the moment of stimulation.

difference was observed between the controls and MCI subjects $(P=.054)$ or between the MCI and AD groups $(P=.336)$. No significant differences were observed in the latencies between the groups (data not shown). Also, no significant interhemispheric differences in P30 were observed $(P=.097)$.

The different groups were discriminated from each other by the ROC curve analysis (Figure 4). The AUC indicated that the discrimination of controls from the MCI and $\mathrm{AD}$ groups is possible based on TMS-induced P30 peak amplitude $\left(\mathrm{AUC}=0.900, P=.024, \mathrm{P} 30_{\text {mean }}\right)$. The optimal cut-off point was found to be $24.5 \mu \mathrm{V}$ (sensitivity of 0.75 and specificity of 0.80 ). Similarly, as the amplitude of the $\mathrm{P} 30$ peak was lower in the $\mathrm{AD}$ group as in the other groups, the ROC curve indicated that the discrimination of the AD group from controls and MCI may be possible $(\mathrm{AUC}=0.882$,

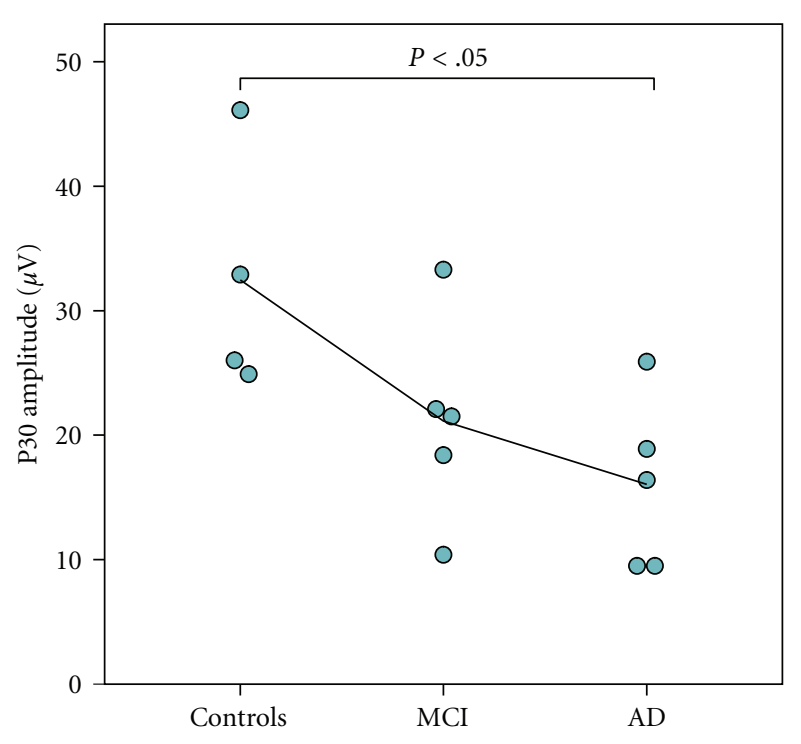

Figure 3: Group-wise P30 amplitudes. The individual values are presented as a mean value of P30 amplitude measured from both hemispheres. Black line represents the group-wise mean value when moving from controls to MCI and AD.

$\left.P=.053, \mathrm{P} 30_{\text {mean }}\right)$. The optimal cut-off point in this case was $20.2 \mu \mathrm{V}$ (sensitivity of 0.80 and specificity of 0.78 ).

If only the discrimination of $\mathrm{AD}$ patients from controls was estimated, the AUC increased to $0.950(P=.027$, P30 mean $)$. The optimal cut-off point was $25.4 \mu \mathrm{V}$ (sensitivity of 0.80 and specificity of 0.75 ). Discrimination of MCI from $\mathrm{AD}$ using ROC curve was found more difficult (AUC $\left.=0.720, P=.251, \mathrm{P} 30_{\text {mean }}\right)$. The optimal cut-off point then was $18.7 \mu \mathrm{V}$ (sensitivity of 0.60 and specificity of $0.60)$. However, the more important discrimination of MCI subjects from controls was found stronger although the AUC was $0.850\left(P=.086, \mathrm{P} 30_{\text {mean }}\right)$. The optimal cut-off point was $25.5 \mu \mathrm{V}$ (sensitivity of 0.75 and specificity of 0.80 ), which was very close to similar as in discriminating $\mathrm{AD}$ patients from controls.

Significant correlations were observed between the P30 amplitude and the dementia scales. An inverse correlation was found between the global CDR and P30 amplitude as well as between CDR-SOB and P30 amplitude (Figure 5). As the global CDR is a classification variable, its correlations with P30 amplitude should be interpreted with care. 


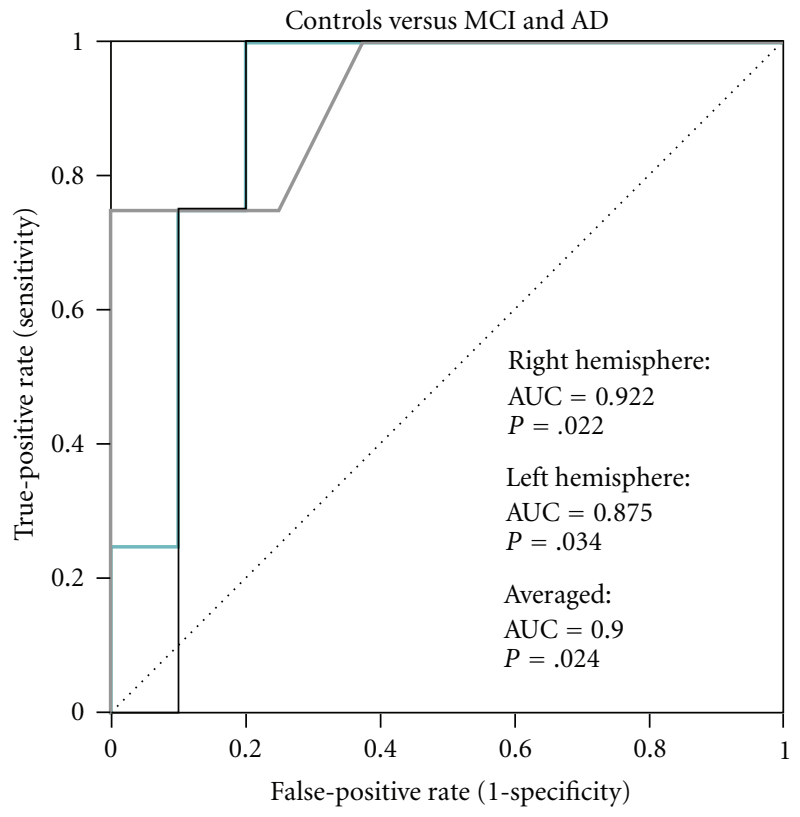

(a)

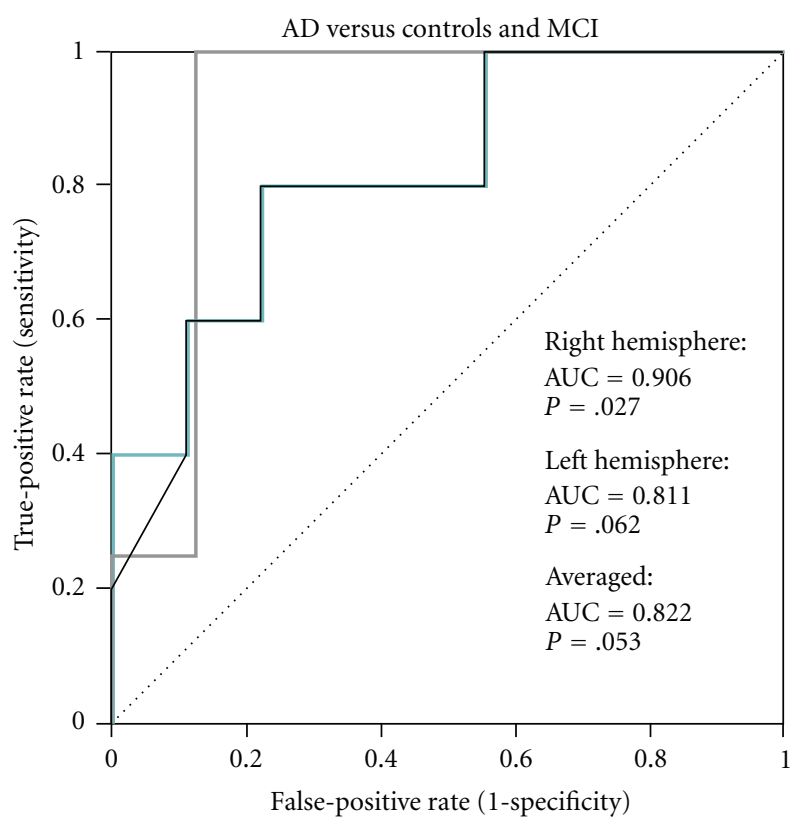

(b)

FIGURE 4: Receiver operating characteristic (ROC) curves for distinguishing (a) controls from MCI and AD, and (b) and AD patients from MCI and control subjects based on TMS-EEG P30. Turquoise line indicates the ROC curve for averaged data, while the grey and black lines indicate ROC curves for the right and left hemisphere, respectively. The area under the ROC curve (AUC) has been given separately for the averaged $\left(\mathrm{P} 30_{\text {mean }}\right)$ and hemispheric data. The asymptotic significance has been indicated with the null hypothesis of AUC $=0.5$ (diagonal line).

TABle 2: Correlation coefficients (Spearman's $\rho$ ) between the P30 amplitude and dementia rating scales.

\begin{tabular}{lccc}
\hline & Mini-mental state examination & Clinical dementia rating-global ${ }^{\dagger}$ & Clinical dementia rating-sum of boxes \\
\hline Left hemisphere & 0.456 & $-0.678^{* *}$ & $-0.788^{* * *}$ \\
Right hemisphere & $0.631^{*}$ & $-0.705^{* *}$ & $-0.849^{* * *}$ \\
P30 mean & $0.537^{*}$ & $-0.698^{* *}$ & $-0.808^{* * *}$ \\
\hline
\end{tabular}

$* P<.05,{ }^{* *} P<.01,{ }^{* * *} P<.001$.

${ }^{\dagger}$ As the global CDR is a classification variable, its correlations with P30 amplitude should be interpreted with care.

Abbreviations:

P30 mean: P30 amplitude, mean of P30 amplitudes on both hemispheres.

A positive correlation was found between the MMSE and P30 amplitude (Table 2).

\section{Discussion}

We have previously shown that TMS-evoked P30 amplitude is reduced in the $\mathrm{AD}$ subjects in the temporoparietal area, ipsilateral to the stimulation side as well as in the contralateral frontocentral cortex corresponding to the sensorimotor area [39]. In the present study, we further investigated our previously published data and found that the discrimination of control subjects from $\mathrm{MCI}$ and $\mathrm{AD}$ subjects may be possible with good sensitivity (Figures 3 and 4). Further, consistently with our hypothesis, we found that there is a significant relation between the commonly used dementia rating scales and the analyzed TMS-EEG response P30 amplitude, when TMS is focused on the M1 with suprathreshold intensity (Figure 5). Our results suggest that the use of TMSEEG in the evaluation of AD and its initial signs could be feasible as distinct changes occur in the measured responses during the development of $\mathrm{AD}$.

The greatest limitation of this study was that the group sizes were small. In spite of that, the findings of the present study showed clearly significant differences between the groups. In the future, these results should be further verified by other studies with larger group sizes. Nevertheless, it was clear that the discrimination of probable mild AD patients and MCI subjects from control subjects seemed feasible. Furthermore, the present study was able to show a correlation between the P30 amplitude of the TMS-EEG and the dementia rating scales (Figure 5, Table 2). Such relation has not been reported earlier. Therefore, it seems that the P30 peak is indeed related to cognitive decline, or perhaps to the developing motor deficits that the $\mathrm{AD}$ patients may exhibit in the advanced stage of the disease [11]. Due to the small sample size, the study may suffer a lack of power to provide reliable answers to its aim, that is, some of the intergroup relations and differences may have been missed. Even with 


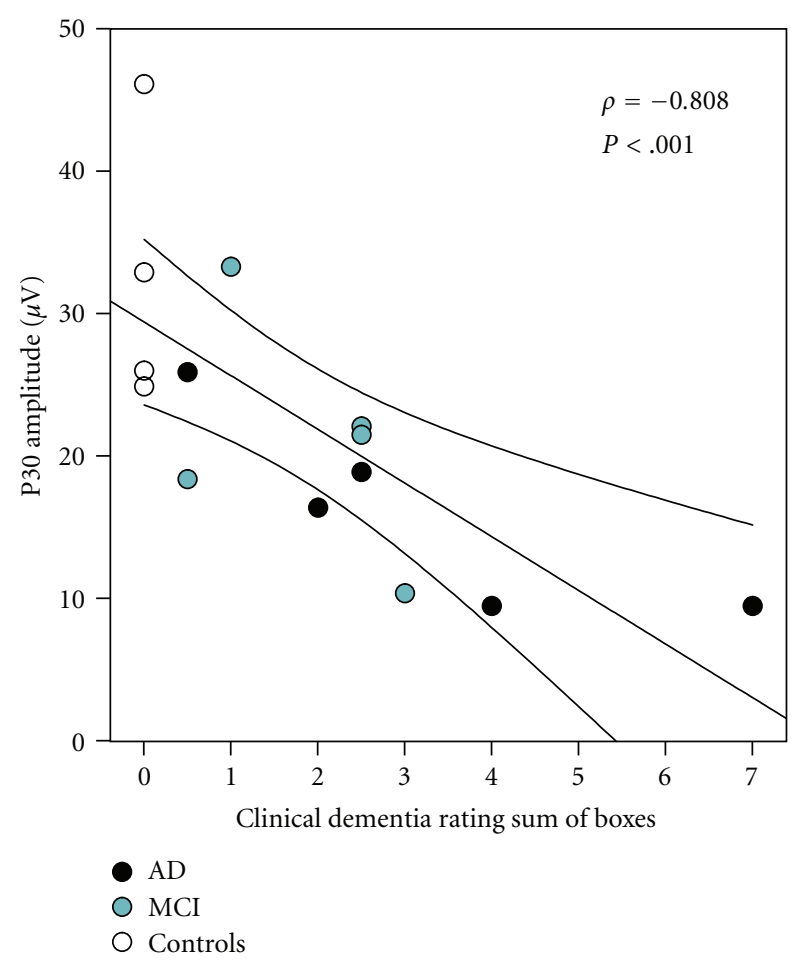

FIGURE 5: Scatter plot indicating the relation between the clinical dementia rating sum of boxes and P30 amplitude (average of each subject's left and right hemisphere measurement, $\mathrm{P} 30_{\text {mean }}$ ). The thin curved lines represent the $95 \%$ confidence intervals for the curve fit.

the small sample size, the found effect size for discriminating controls from mild $\mathrm{AD}$ patients based on the $\mathrm{P} 30_{\text {mean }}$ was large (Cohen's $d>0.8$ ), as was the effect size for the controls and MCI difference (Cohen's $d>0.8$ ). A medium size effect was observed between the MCI and AD (Cohen's $d>0.5$ ). However, the statistical significance of those comparisons was too weak in the last two cases. This encourages further studies with larger sample sizes.

As opposed to the localized (single-channel) P30 responses reported in the present study, our previous study investigated the global mean field power (GMFP) of the P30 peak [39]. Hence, for comparison, the global mean field power (GMFP) was computed for the P30 response peaks $\left(\mathrm{P} 30_{\mathrm{GMFP}}\right)$ [49]. We found that the $\mathrm{P} 30_{\mathrm{GMFP}}$ correlated strongly with the single-channel $\mathrm{P} 30_{\text {mean }}(\rho=0.810, P<$ $.001)$. This correlation is affected by the differences in the P30 spread between the groups, and hence the correlation may not be an ideal indicator for similar behavior. In the discrimination of the different groups, $\mathrm{P} 30_{\mathrm{GMFP}}$ was weaker than the single-channel P30 mean, that is, in the ROC analysis, controls were not discriminated as easily from the MCI and $\mathrm{AD}(\mathrm{AUC}=0.775, P=.120$ ) or the $\mathrm{AD}$ group from $\mathrm{MCI}$ and control groups (AUC $=0.756, P=.125$ ). Furthermore, the P30 $0_{\text {GMFP }}$ exhibited some correlations with the dementia scales $\left(\mathrm{P} 30_{\mathrm{GMFP}}\right.$ versus MMSE, $\rho=0.311, P=.279 ; \mathrm{P} 30_{\mathrm{GMFP}}$ versus $\mathrm{CDR}, \rho=-0.515, P=.060$; $\mathrm{P} 30_{\mathrm{GMFP}}$ versus $\mathrm{CDR}-$ SOB, $\rho=-0.755, P=.002$ ). Therefore, to us it seems that the localized P30 amplitude is more sensitive in observing cognitive decline as compared to global field values. This finding may be influenced by the modified spread of the P30 component in $\mathrm{AD}$, which was observed in our previous study, and which affects the GMFP [39].

The CDR is a standard assessment tool that yields global and CDR-SOB scores. The global CDR score is often used to stage dementia severity [45]. However, the CDR-SOB score is a more detailed general index and is more sensitive in assessing mild dementia [50-52]. Based on the CDR-SOB, the $\mathrm{AD}$ patients in this study had very mild or mild dementia [52]. In agreement with our hypothesis, we found significant correlations between the P30 amplitude and the dementia scales (Table 2). As the CDR-SOB is a detailed and one of the most used dementia scales, it also related best to the P30 amplitude as was seen from their strong correlation (Figure 5). Also, the other applied dementia scales correlated with P30. Therefore, it seems that P30 indeed relates to the severity of the $\mathrm{AD}$ even in a mild stage of the disease as the patients were in the present study. Since our recent study showed some effect of cognitive decline on the N100 [39], we analyzed the N100 response from the TMS-EEG at the vertex for comparison with the P30 amplitude. We found that none of the dementia scales correlated significantly with the N100 amplitude (N100 versus MMSE, $\rho=-0.029$; N100 versus CDR, $\rho=0.333$; N100 versus CDR-SOB, $\rho=$ 0.177). Therefore, P30 appears more specific than N100 in identifying cognitive decline with TMS-EEG. The reason for this may be that TMS-induced N100 response also includes an auditory component, which does not affect P30 [42].

Currently, the origin of $\mathrm{P} 30$ is not precisely described. It has been suggested to originate from the ipsilateral sensorimotor/premotor cortex border [41] or the ipsilateral supplementary motor area [53]. As discussed by Mäki and Ilmoniemi, the P30 may not reflect activation directly at the location activated by the stimulus. However, it may still reflect the degree of excitation in M1 [33]. Bonato et al. showed that P30 vanishes if the activation of M1 is induced with TMS coil oriented nonoptimally, which supports the idea that P30 is descriptive of cortical activation related to M1 excitation [40]. Considering the findings of the present study, the decrease in P30 in AD as compared to healthy controls may reflect impaired cortical activation in response to $\mathrm{M} 1$ activation. However, if we consider the earlier findings relating MEPs with P30, the P30 measured in the present study would not directly relate to motor activation, as the earlier reports have indicated a positive correlation between the two [33, 40]. In our earlier study [39], we showed that the correlation between the induced MEPs and P30 exhibits a nonsignificant negative trend when correlated over different patient groups $(\rho=-0.224, P=.441)$. When comparing the mean values of MEP amplitudes, the controls exhibit the lowest mean MEP amplitude of $1.0 \mathrm{mV}$ while showing the highest P30 amplitude. Instead, the highest mean MEP amplitude of $2.9 \mathrm{mV}$ was in the $\mathrm{AD}$ group with the lowest P30 amplitude (Figure 3 ). This suggests that P30 is not only related to the excitation of M1, but also other mechanisms may influence it when AD progresses. Ferreri et al. [38] suggested that $\mathrm{P} 30$ may be connected to $\mathrm{GABA}_{\mathrm{A}}$ receptors, provided that P30 modulation is related to fast inhibitory 
postsynaptic potentials. Hence, considering the earlier findings suggesting reduction in $\mathrm{GABA}_{\mathrm{A}}$-mediated SICI of $\mathrm{AD}$ patients $[3,18-20]$, the reduction in P30 amplitude in relation to cognitive decline could be explained by possibly affected $\mathrm{GABA}_{\mathrm{A}}$ activity in $\mathrm{AD}$ patients, even if the effect is not directly caused by the disease. However, based on the present study, we cannot reveal the mechanism affecting P30, and future studies are required.

As the P30 has been suggested to relate to motor function/control in response to TMS of the M1 [33, 40], it is obvious that it may also be affected by the motor function impairment related to the development of AD. Furthermore, as the connectivity of the $\mathrm{AD}$ patients is also impaired, the subcortical contributor to the P30 may suffer leading to a decrease in P30 amplitude (Figure 5). The indications of P30 decrease are already present in the MCI subjects (Figure 4) although they are insignificant $(P=.054)$ in the present study, likely due to the low number of subjects. One possibility is that the $\mathrm{P} 30$ decline in $\mathrm{AD}$ is a result of the missing connections or cortical atrophy, and the actual function, which is still unclear, is related to the cortical hyperexcitability $[19,54]$ or cholinergic dysfunction leading to disinhibition $[17-19,55]$. Hence, we will further study on how the dementia symptoms relate to the connectivity and the components of the TMS-EEG in our future studies with larger material.

We cannot rule out the possible effect of medication in our findings, as the AD patients were on cholinesterase inhibitors. However, in our previous study [39], we found that the later part of the TMS-EEG response appeared partly similar to that of the controls, meaning that not the entire TMS-EEG response is influenced by either the AD or the medication, or that the cholinesterase inhibitors are compensating for some of the changes. The effect of cholinesterase inhibitors on the motor cortex excitability has been studied earlier. Korchounov et al. [56] found that the MTs or SPs were not affected by the acetylcholinesterase inhibitor in healthy subjects, while the intracortical inhibition and facilitation were affected between 2 and 6 hours of the medication intake. The motor cortex disinhibition in $\mathrm{AD}$ has been shown to recover partly by the use of cholinesterase inhibitors [55]. Studying the effect of cholinesterase inhibitors on the TMSEEG response of healthy subjects, possibly in combination with paradigms such as SAI or paired-pulse TMS, could help to understand the origin of P30.

\section{Conclusions}

We found differences in TMS-induced P30-component amplitude between the controls and AD patients, indicating impaired/altered connectivity in AD. In addition, we found that the cognitive decline correlated with the P30 amplitude. Further investigations with larger sample sizes are needed to support our conclusion that TMS-EEG could be a potential noninvasive biomarker for identifying MCI and AD subjects and separating those from healthy population, and for identifying connectivity changes occurring during the development of AD.

\section{Conflict of Interests}

Professor J. Karhu works part time as Chief Medical Officer in Nexstim Ltd., the manufacturer of navigated brain stimulation instruments.

\section{Acknowledgment}

This study was supported by EVO (5772739) Grant from Kuopio University Hospital.

\section{References}

[1] W. A. Rocca, A. Hofman, C. Brayne et al., "Frequency and distribution of Alzheimer's disease in Europe: a collaborative study of 1980-1990 prevalence findings. The EURODEMPrevalence Research Group," Annals of Neurology, vol. 30, no. 3, pp. 381-390, 1991.

[2] M. M. Mesulam, E. J. Mufson, A. I. Levey, and B. H. Wainer, "Cholinergic innervation of cortex by the basal forebrain: cytochemistry and cortical connections of the septal area, diagonal band nuclei, nucleus basalis (substantia innominata), and hypothalamus in the rhesus monkey," Journal of Comparative Neurology, vol. 214, no. 2, pp. 170-197, 1983.

[3] A. Martorana, A. Stefani, M. G. Palmieri et al., "L-dopa modulates motor cortex excitability in Alzheimer's disease patients," Journal of Neural Transmission, vol. 115, no. 9, pp. 1313-1319, 2008.

[4] J. A. Hardy, D. M. A. Mann, P. Wester, and B. Winblad, "An integrative hypothesis concerning the pathogenesis and progression of Alzheimer's disease," Neurobiology of Aging, vol. 7, no. 6, pp. 489-502, 1986.

[5] C. L. Grady, M. L. Furey, P. Pietrini, B. Horwitz, and S. I. Rapoport, "Altered brain functional connectivity and impaired short-term memory in Alzheimer's disease," Brain, vol. 124, no. 4, pp. 739-756, 2001.

[6] J. L. Price and J. C. Morris, "Tangles and plaques in nondemented aging and 'preclinical' alzheimer's disease," Annals of Neurology, vol. 45, no. 3, pp. 358-368, 1999.

[7] R. C. Petersen, G. E. Smith, R. J. Ivnik et al., "Apolipoprotein E status as a predictor of the development of Alzheimer's disease in memory-impaired individuals," Journal of the American Medical Association, vol. 273, no. 16, pp. 1274-1278, 1995.

[8] J. C. Morris, M. Storandt, J. P. Miller et al., "Mild cognitive impairment represents early-stage Alzheimer disease," Archives of Neurology, vol. 58, no. 3, pp. 397-405, 2001.

[9] R. C. Petersen, "Mild cognitive impairment: transition between aging and Alzheimer's disease," Neurologia, vol. 15, no. 3, pp. 93-101, 2000.

[10] J. Y. Chen, Y. Stern, M. Sano, and R. Mayeux, "Cumulative risks of developing extrapyramidal signs, psychosis, or myoclonus in the course of Alzheimer's disease," Archives of Neurology, vol. 48, no. 11, pp. 1141-1143, 1991.

[11] G. McKhann, D. Drachman, and M. Folstein, "Clinical diagnosis of Alzheimer's disease: report of the NINCDS-ADRDA work group under the auspices of Department of Health and Human Services Task Force on Alzheimer's disease," Neurology, vol. 34, no. 7, pp. 939-944, 1984.

[12] D. S. Knopman, S. T. DeKosky, J. L. Cummings et al., "Practice parameter: diagnosis of dementia (an evidence-based review): report of the quality standards subcommittee of the american academy of neurology," Neurology, vol. 56, no. 9, pp. 1143$1153,2001$. 
[13] R. C. A. Pearson, M. M. Esiri, and R. W. Hiorns, "Anatomical correlates of the distribution of the pathological changes in the neocortex in Alzheimer disease," Proceedings of the National Academy of Sciences of the United States of America, vol. 82, no. 13, pp. 4531-4534, 1985.

[14] J. Rogers and J. H. Morrison, "Quantitative morphology and regional and laminar distributions of senile plaques in Alzheimer's disease," Journal of Neuroscience, vol. 5, no. 10, pp. 2801-2808, 1985.

[15] G. Alagona, R. Bella, R. Ferri et al., "Transcranial magnetic stimulation in Alzheimer disease: motor cortex excitability and cognitive severity," Neuroscience Letters, vol. 314, no. 1-2, pp. 57-60, 2001.

[16] M. De Carvalho, A. De Mendonça, P. C. Miranda, C. Garcia, and M. De Lourdes Sales Luís, "Magnetic stimulation in Alzheimer's disease," Journal of Neurology, vol. 244, no. 5, pp. 304-307, 1997.

[17] V. Di Lazzaro, F. Pilato, M. Dileone et al., "In vivo cholinergic circuit evaluation in frontotemporal and Alzheimer dementias," Neurology, vol. 66, no. 7, pp. 1111-1113, 2006.

[18] V. Di Lazzaro, A. Oliviero, P. A. Tonali et al., "Noninvasive in vivo assessment of cholinergic cortical circuits in $\mathrm{AD}$ using transcranial magnetic stimulation," Neurology, vol. 59, no. 3, pp. 392-397, 2002.

[19] V. Di Lazzaro, A. Oliviero, F. Pilato et al., "Motor cortex hyperexcitability to transcranial magnetic stimulation in Alzheimer's disease," Journal of Neurology, Neurosurgery and Psychiatry, vol. 75, no. 4, pp. 555-559, 2004.

[20] A. Martorana, F. Mori, Z. Esposito et al., "Dopamine modulates cholinergic cortical excitability in Alzheimer's disease patients," Neuropsychopharmacology, vol. 34, no. 10, pp. 2323 2328, 2009.

[21] A. Perretti, D. Grossi, N. Fragassi et al., "Evaluation of the motor cortex by magnetic stimulation in patients with Alzheimer disease," Journal of the Neurological Sciences, vol. 135, no. 1, pp. 31-37, 1996.

[22] V. Di Lazzaro, A. Oliviero, F. Pilato et al., "Neurophysiological predictors of long term response to AChE inhibitors in AD patients," Journal of Neurology, Neurosurgery and Psychiatry, vol. 76, no. 8, pp. 1064-1069, 2005.

[23] K. Sakuma, T. Murakami, and K. Nakashima, "Short latency afferent inhibition is not impaired in mild cognitive impairment," Clinical Neurophysiology, vol. 118, no. 7, pp. 1460 1463, 2007.

[24] R. Nardone, J. Bergmann, M. Kronbichler et al., "Abnormal short latency afferent inhibition in early Alzheimer's disease: a transcranial magnetic demonstration," Journal of Neural Transmission, vol. 115, no. 11, pp. 1557-1562, 2008.

[25] V. Di Lazzaro, A. Oliviero, P. Profice et al., "Muscarinic receptor blockade has differential effects on the excitability of intracortical circuits in the human motor cortex," Experimental Brain Research, vol. 135, no. 4, pp. 455-461, 2000.

[26] H. Tokimura, V. Di Lazzaro, Y. Tokimura et al., "Short latency inhibition of human hand motor cortex by somatosensory input from the hand," Journal of Physiology, vol. 523, no. 2, pp. 503-513, 2000.

[27] R. Mariorenzi, F. Zarola, M. D. Caramia, C. Paradiso, and P. M. Rossini, "Non-invasive evaluation of central motor tract excitability changes following peripheral nerve stimulation in healthy humans," Electroencephalography and Clinical Neurophysiology, vol. 81, no. 2, pp. 90-101, 1991.

[28] M. L. Gilmor, J. D. Erickson, H. Varoqui et al., "Preservation of nucleus basalis neurons containing choline acetyltransferase and the vesicular acetylcholine transporter in the elderly with mild cognitive impairment and early Alzheimer's disease," Journal of Comparative Neurology, vol. 411, no. 4, pp. 693-704, 1999.

[29] S. Salloway, S. Ferris, A. Kluger et al., "Efficacy of donepezil in mild cognitive impairment: a randomized placebo-controlled trial," Neurology, vol. 63, no. 4, pp. 651-657, 2004.

[30] A. J. Saykin, H. A. Wishart, L. A. Rabin et al., "Cholinergic enhancement of frontal lobe activity in mild cognitive impairment," Brain, vol. 127, no. 7, pp. 1574-1583, 2004.

[31] E. J. Mufson, S. Y. Ma, J. Dills et al., "Loss of basal forebrain p75 immunoreactivity in subjects with mild cognitive impairment and Alzheimer's disease," Journal of Comparative Neurology, vol. 443, no. 2, pp. 136-153, 2002.

[32] U. Ziemann, S. Lönnecker, B. J. Steinhoff, and W. Paulus, "Effects of antiepileptic drugs on motor cortex excitability in humans: a transcranial magnetic stimulation study," Annals of Neurology, vol. 40, no. 3, pp. 367-378, 1996.

[33] H. Mäki and R. J. Ilmoniemi, "The relationship between peripheral and early cortical activation induced by transcranial magnetic stimulation," Neuroscience Letters, vol. 478, no. 1, pp. 24-28, 2010.

[34] T. Raij, J. Karhu, D. Kičić et al., "Parallel input makes the brain run faster," NeuroImage, vol. 40, no. 4, pp. 1792-1797, 2008.

[35] S. Komssi, H. J. Aronen, J. Huttunen et al., "Ipsi- and contralateral EEG reactions to transcranial magnetic stimulation," Clinical Neurophysiology, vol. 113, no. 2, pp. 175-184, 2002.

[36] M. Massimini, F. Ferrarelli, R. Huber, S. K. Esser, H. Singh, and G. Tononi, "Neuroscience: breakdown of cortical effective connectivity during sleep," Science, vol. 309, no. 5744, pp. 2228-2232, 2005.

[37] M. Rosanova, A. Casali, V. Bellina, F. Resta, M. Mariotti, and M. Massimini, "Natural frequencies of human corticothalamic circuits," Journal of Neuroscience, vol. 29, no. 24, pp. 76797685, 2009.

[38] F. Ferreri, P. Pasqualetti, S. Määttä et al., "Human brain connectivity during single and paired pulse transcranial magnetic stimulation," NeuroImage, vol. 54, no. 1, pp. 90-102, 2011.

[39] P. Julkunen, A. M. Jauhiainen, S. Westerén-Punnonen et al., "Navigated TMS combined with EEG in mild cognitive impairment and Alzheimer's disease: a pilot study," Journal of Neuroscience Methods, vol. 172, no. 2, pp. 270-276, 2008.

[40] C. Bonato, C. Miniussi, and P. M. Rossini, "Transcranial magnetic stimulation and cortical evoked potentials: a TMS/EEG co-registration study," Clinical Neurophysiology, vol. 117, no. 8, pp. 1699-1707, 2006.

[41] S. K. Esser, R. Huber, M. Massimini, M. J. Peterson, F. Ferrarelli, and G. Tononi, "A direct demonstration of cortical LTP in humans: a combined TMS/EEG study," Brain Research Bulletin, vol. 69, no. 1, pp. 86-94, 2006.

[42] V. Nikouline, J. Ruohonen, and R. J. Ilmoniemi, "The role of the coil click in TMS assessed with simultaneous EEG," Clinical Neurophysiology, vol. 110, no. 8, pp. 1325-1328, 1999.

[43] T. Paus, P. K. Sipilä, and A. P. Strafella, "Synchronization of neuronal activity in the human primary motor cortex by transcranial magnetic stimulation: an EEG study," Journal of Neurophysiology, vol. 86, no. 4, pp. 1983-1990, 2001.

[44] P. Julkunen, L. Säisänen, N. Danner et al., "Comparison of navigated and non-navigated transcranial magnetic stimulation for motor cortex mapping, motor threshold and motor evoked potentials," NeuroImage, vol. 44, no. 3, pp. 790-795, 2009. 
[45] C. P. Hughes, L. Berg, and W. L. Danziger, "A new clinical scale for the staging of dementia," British Journal of Psychiatry, vol. 140, no. 6, pp. 566-572, 1982.

[46] M. F. Folstein, S. E. Folstein, and P. R. McHugh, “'Mini mental state. A practical method for grading the cognitive state of patients for the clinician," Journal of Psychiatric Research, vol. 12, no. 3, pp. 189-198, 1975.

[47] S. Gauthier, B. Reisberg, M. Zaudig et al., "Mild cognitive impairment," Lancet, vol. 367, no. 9518, pp. 1262-1270, 2006.

[48] F. Awiszus, "Chapter 2 TMS and threshold hunting," Supplements to Clinical Neurophysiology, vol. 56, pp. 13-23, 2003.

[49] D. Lehmann and W. Skrandies, "Reference-free identification of components of checkerboard-evoked multichannel potential fields," Electroencephalography and Clinical Neurophysiology, vol. 48, no. 6, pp. 609-621, 1980.

[50] L. Berg, J. P. Miller, M. Storandt et al., "Mild senile dementia of the Alzheimer type: 2. Longitudinal assessment," Annals of Neurology, vol. 23, no. 5, pp. 477-484, 1988.

[51] C. A. Lynch, C. Walsh, A. Blanco et al., "The clinical dementia rating sum of box score in mild dementia," Dementia and Geriatric Cognitive Disorders, vol. 21, no. 1, pp. 40-43, 2006.

[52] S. E. O’Bryant, S. C. Waring, C. M. Cullum et al., "Staging dementia using clinical dementia rating scale sum of boxes scores: a Texas Alzheimer's research consortium study," Archives of Neurology, vol. 65, no. 8, pp. 1091-1095, 2008.

[53] V. Litvak, S. Komssi, M. Scherg et al., "Artifact correction and source analysis of early electroencephalographic responses evoked by transcranial magnetic stimulation over primary motor cortex," NeuroImage, vol. 37, no. 1, pp. 56-70, 2007.

[54] F. Ferreri, F. Pauri, P. Pasqualetti, R. Fini, G. Dal Forno, and P. M. Rossini, "Motor cortex excitability in Alzheimer's disease: a transcranial magnetic stimulation study," Annals of Neurology, vol. 53, no. 1, pp. 102-108, 2003.

[55] J. Liepert, K. J. Bar, U. Meske et al., "Motor cortex disinhibition in Alzheimer's disease," Clinical Neurophysiology, vol. 112, no. 8, pp. 1436-1441, 2001.

[56] A. Korchounov, T. V. Ilic, T. Schwinge, and U. Ziemann, "Modification of motor cortical excitability by an acetylcholinesterase inhibitor," Experimental Brain Research, vol. 164, no. 3, pp. 399-405, 2005.

[57] E. Niskanen, P. Julkunen, L. Säisänen, R. Vanninen, P. Karjalainen, and M. Könönen, "Group-level variations in motor representation areas of thenar and anterior tibial muscles: navigated transcranial magnetic stimulation study," Human Brain Mapping, vol. 31, no. 8, pp. 1272-1280, 2010. 


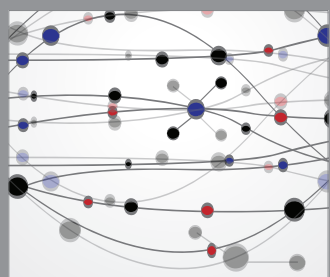

The Scientific World Journal
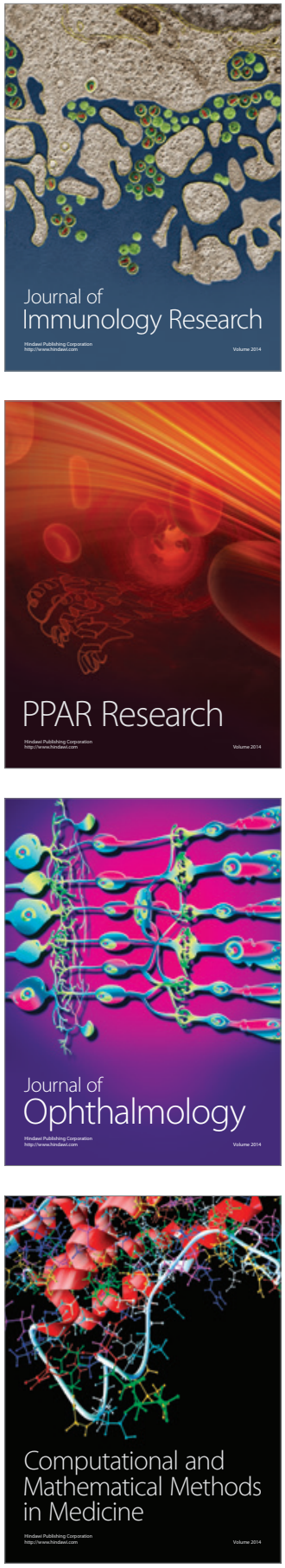

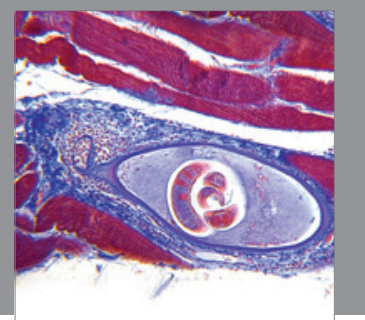

Gastroenterology

Research and Practice
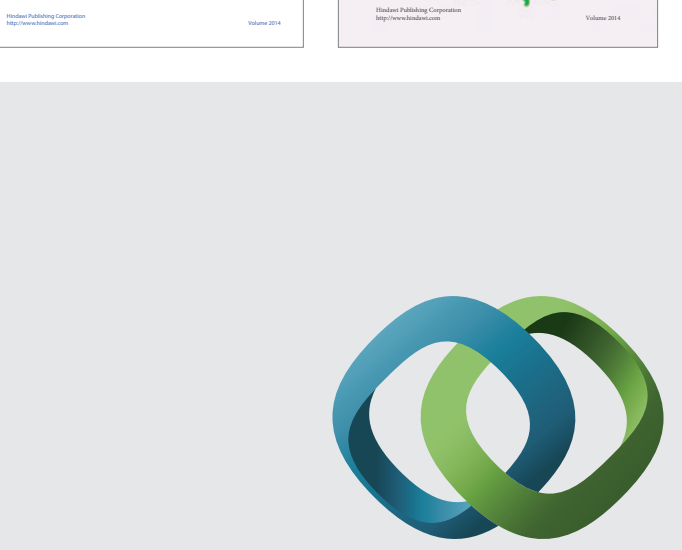

\section{Hindawi}

Submit your manuscripts at

http://www.hindawi.com
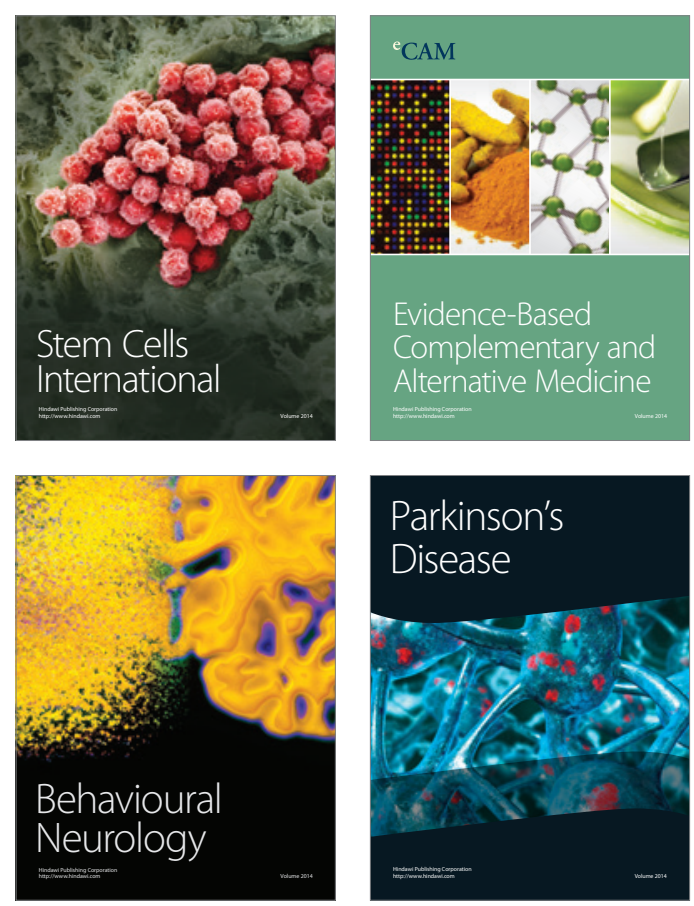

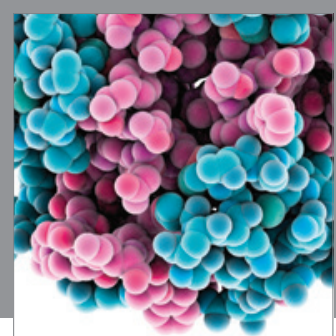

Journal of
Diabetes Research

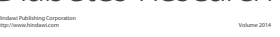

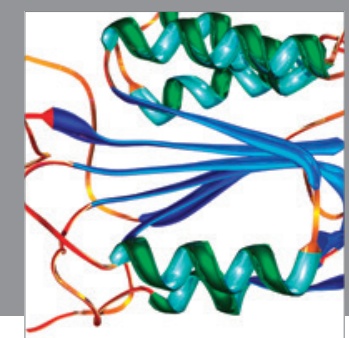

Disease Markers
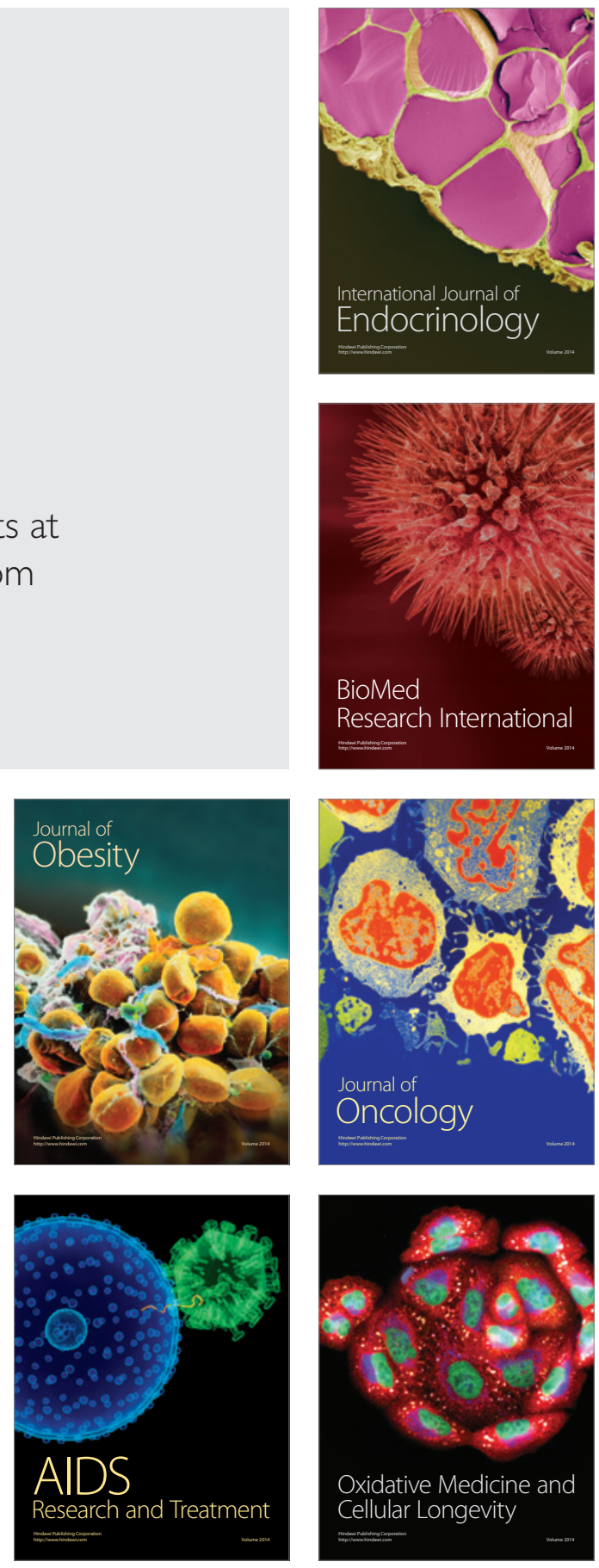